\title{
The Disparate Effects of Epinephrine and Norepinephrine on Hyperglycemia in Cardiovascular Surgery
}

\author{
Daniel Phadke, BS, Jared P. Beller, MD, Curt Tribble, MD \\ Division of Thoracic and Cardiovascular Surgery, Department of Surgery, University of Virginia, Charlottesville, Virginia, USA
}

\section{ABSTRACT}

Hyperglycemia is a metabolic derangement that frequently develops after cardiovascular surgery. The perioperative administration of inotropic and vasoactive agents, such as epinephrine and norepinephrine, are common in the management of cardiac surgery patients and are known to contribute to the development of postoperative hyperglycemia. We hypothesized that hemodynamic support with epinephrine exacerbates postoperative hyperglycemia to a greater degree than does treatment with norepinephrine. This literature review outlines the mechanisms by which epinephrine and norepinephrine alter glucose homeostasis, while highlighting the significant differences in their effects on hepatic glucose mobilization and peripheral glucose utilization. This review suggests that the use of epinephrine exacerbates postoperative hyperglycemia to a greater degree than does norepinephrine.

\section{INTRODUCTION}

Hyperglycemia is a metabolic derangement commonly encountered and treated in everyday medical practice and is associated with increased rates of morbidity and mortality in both medical and surgical settings [Gerstein 2014; Girish 2014; Ito 2014; Mulla 2014; Reddy 2014]. Disorders of glucose homeostasis have downstream effects on systemic and cardiac-specific metabolic and inflammatory states, which have been associated with adverse clinical outcomes [Furnary 2003]. Furthermore, abnormal glucose levels have been found to be an independent risk factor for adverse cardiovascular events, both in patients requiring coronary revascularization and those undergoing other cardiovascular procedures [Bartnik 2004a, Bartnik 2004b].

After cardiac surgery, postoperative hyperglycemia is found in up to $80 \%$ of patients. Effective glycemic control is especially important in these patients because of the significant association between stress hyperglycemia and adverse outcomes. In the postoperative state, hyperglycemia contributes to direct cellular toxicity, increased

Received March 23, 2018; received in revised form May 1, 2018; accepted May 5, 2018 .

Correspondence: Curtis G. Tribble, MD, Department of Surgery, University of Virginia School of Medicine, PO Box 800300, Charlottesville, VA 22908; 1-434-924-2145 (e-mail: CGT2E@virginia.edu). apoptosis, immunologic modulation, vascular alteration, and impairment in wound healing [Girish 2014].

Most cardiac surgery patients are managed with intravenous inotropes in the early postoperative period. Although there are various institutional protocols and individual preferences, common first-line agents include epinephrine and norepinephrine. Although these agents are known to play a role in the development of postoperative hyperglycemia, their different effects on hyperglycemia are currently underrecognized and rarely discussed. The differences in the specific mechanisms of each catecholamine's action may play an important role in how glucose is regulated in the physiologically stressful postoperative state. We hypothesized that the use of norepinephrine in the perioperative management of cardiac surgery patients is associated with improved glucose control when compared to epinephrine. The purpose of this review is to outline the current evidence to support that hypothesis.

\section{MATERIALS AND METHODS}

Data and information in this review were compiled by using the NCBI's PubMed electronic database using the following search queries: "epinephrine", "norepinephrine", "glucose metabolism", "cardiac surgery", and "post-operative hyperglycemia". Only peer-reviewed material was considered for inclusion in this review. Unpublished material, studies in preparation for publication, and any otherwise unpublished communications were not included.

\section{NORMAL GLUCOSE HOMEOSTASIS}

An intricate network of hormones and neuropeptides tightly regulate blood glucose concentrations under physiologic conditions. These compounds are released primarily from the brain, pancreas, liver, intestine, adipose tissue, and muscle. This complex system allows for tight regulation of serum glucose levels under normal conditions. For example, a decrease in plasma glucose as small as $20 \mathrm{mg} / \mathrm{dL}$ (from 90 to $70 \mathrm{mg} / \mathrm{dL}$ ) will suppress pancreatic insulin release and decrease the uptake of glucose in certain parts of the brain [Gerich 1988]. This results in activation of the sympathetic nervous system and leads to the secretion of counterregulatory hormones like glucagon, catecholamines, cortisol, and growth hormone [Gerich 1988]. These substances will act to increase the release of glucose into the plasma in order to achieve a state of normoglycemia. 
EPINEPHRINE

\section{Effect of Epinephrine on Glucose Regulation}

Epinephrine is a naturally occurring catecholamine released in response to a number of physiologic stimuli. These stimuli are generally grouped into one of 4 categories: physical, psychological, social, and cardiovascular/metabolic [Kvetnansky 2009]. The characterization of epinephrine and its effects began in the nineteenth century when the first reports of the cardiovascular effects of a then unknown adrenal extract were published [Oliver 1895]. Shortly thereafter, the compound itself was isolated and its structure identified [Abel 1897; Takamine 1901].

A relationship between epinephrine and glucose metabolism has long been appreciated, but over time, the characterization of the specific mechanisms of its action has developed in a stepwise fashion. Initially, a rise in blood glucose in response to epinephrine was attributed to increased hepatic glycogen breakdown. This early understanding of the effects of epinephrine on glucose regulation relied on studies that utilized supraphysiologic serum concentrations of this catecholamine. This approach limited a complete understanding of epinephrine's specific mechanism of action because the resultant hyperglycemia was mediated via both alpha- and beta-adrenergic pathways [Antonis 1967]. Subsequent experiments helped to show that, in addition to its powerful influence on hepatic glucose production, epinephrine also exerts its influence through other routes, including attenuation of peripheral utilization of glucose and enhanced gluconeogenesis [Sherwin 1984].

In addition, epinephrine was found to have significant effects on glucose regulation via the potentiation of responses to other stress hormones. The synergism between epinephrine and both glucagon and cortisol contributes to the profound systemic perturbations in glycemic control that follow physiologic stresses such as cardiopulmonary bypass and major surgery. Eigler et al demonstrated this principle in a canine model in which isolated infusions of glucagon, epinephrine, or cortisol resulted in only mild or insignificant elevations in plasma glucose concentration [Eigler 1979]. However, the increase in plasma glucose produced by the combined infusion of any 2 of these counterregulatory hormones was $50 \%$ to $215 \%$ greater than the sum of the respective individual infusions. In each case, any infusion of these other hormones, in combination with epinephrine, produced greater increases in plasma glucose concentration than individual infusions of each hormone or combinations of them when not infused with epinephrine. All 3 hormones together produced an increase in plasma glucose of $144 \mathrm{mg} /$ dL. These data suggest that changes in glucose metabolism seen in stress hyperglycemia, a period in which several of these counterregulatory hormones are elevated, are a consequence of the synergy between epinephrine and other hormones [Eigler 1979].

\section{Epinephrine and Hepatic Glucose Production}

Epinephrine exerts its influence on hepatic glucose production through the binding of $\mathrm{G}$ protein-coupled adrenergic receptors in the liver, which leads to increased cyclic adenosine monophosphate (cAMP) and stimulation of protein kinase A followed by activation of phosphorylase kinases [Rui 2014]. The initial studies on the kinetics of these interactions utilized infusions of epinephrine and glucagon in a canine model in which insulin levels were held constant [Dufour 2009]. These studies led to the understanding that a certain refractoriness to stable levels of glucose rapidly develops, whereas additional elevations of glucose levels could be achieved through either an additional stimulus or an alteration in the concentration of the hormones being studied. As a result of these early studies, as well as more contemporary research, 2 conclusions can be drawn: (1) that the steady state achieved is not due to depletion of glycogen or substrate for gluconeogenesis, but rather is due to an alteration in sensing, and (2) that a noncAMP-mediated pathway for epinephrine's influence may exist [Sacca 1978].

The initial increase in hepatic-derived serum glucose is mediated first through glycogenolysis and subsequently through increases in the rate of gluconeogenesis [Dufour 2009]. Initially, gluconeogenesis remains at a basal rate, and epinephrine exerts its effects primarily through enhanced breakdown of glycogen. However, after approximately 60 minutes of infusion of epinephrine, glycogenolysis slows and gluconeogenesis rapidly increases, leading to a 4:1 ratio in favor of production (gluconeogenesis) over breakdown (glycogenolysis). Thus, epinephrine has a profound effect on net glucose production by the liver, both through glycogenolysis and gluconeogenesis, but the relative contributions of these pathways vary over time.

\section{Epinephrine's Interaction with Insulin and Peripheral Glucose Uptake}

Insulin serves to stimulate glucose utilization and its removal from the bloodstream, whereas epinephrine is known to attenuate the effects of insulin. Early work in this arena was carried out by Walaas and colleagues, who created an isolated rat diaphragm preparation and were the first to demonstrate epinephrine's ability to block insulin-stimulated muscle uptake in vitro [Walaas 1950]. The key observation in their study was that, in the presence of epinephrine, glucose uptake by muscle was inhibited, which resulted in diminished glycogen deposition when compared to controls. However, these researchers failed to directly link these effects to interactions of epinephrine with insulin, which, it is now known, mediates this epinephrine-induced attenuation of glucose uptake.

In addition to epinephrine's inhibition of the peripheral effects of insulin, these hormones interact in the liver as well. First shown in an animal model, these findings were later corroborated in humans in a euglycemic clamp model, which demonstrated conclusively that insulin's effects were blunted by epinephrine [Eigler 1979; Deibert 1980]. In both of the major sites of glucose uptake during hyperinsulinemic states, peripheral muscle and liver, a dramatic, direct inhibitory effect of epinephrine was observed. Even during hyperinsulinemia, epinephrine retains its ability to stimulate systemic glucose release via the liver. Interestingly, these interactions are all significantly attenuated, if not reversed, by propranolol, a 
nonselective beta-blocker, suggesting that these effects are driven primarily through epinephrine's beta-adrenergic stimulation [Deibert 1980]. Epinephrine attenuates the action of insulin, both at the site of glucose uptake peripherally and by stimulation of glucose utilization and production in the liver.

\section{NOREPINEPHRINE}

\section{Effect of Norepinephrine on Glucose Regulation}

Norepinephrine was first discovered in 1946 by Ulf von Euler as the neurotransmitter in sympathetic nerves and the precursor of epinephrine [von Euler 1946]. In states of stress, circulating levels of norepinephrine can increase up to 4 times the basal levels [Cryer 1980]. However, even at this elevated level, there is a negligible effect of norepinephrine on glucose metabolism [Hjemdahl 1979]. Neural-derived norepinephrine plays a larger role in systemic and hepatic glucose regulation when compared to circulating norepinephrine [Biggers 1989]. Early studies showed that, with splanchnic nerve stimulation, neural-derived norepinephrine can increase hepatic glucose production [Lautt 1983; Edwards 1972; Hatmann 1982]. Contemporary evidence further supports this mechanism, because it has been shown that various hepatic-resident cell lines have adrenergic innervation [Nobin 1977].

\section{Norepinephrine and Hepatic Glucose Production}

Norepinephrine in circulation, as well as norepinephrine released under the influence of hepatic adrenergic innervation, will stimulate gluconeogenesis. In mammals, the effects of norepinephrine on gluconeogenesis are dose dependent;

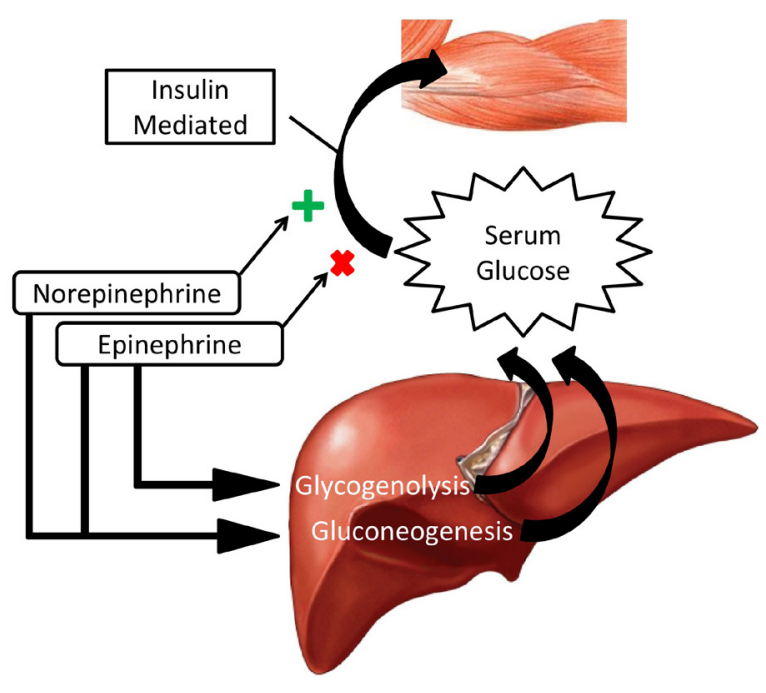

Pathophysiology of catecholamine-regulated glucose modulation. Both norepinephrine and epinephrine increase hepatic glucose output via gluconeogenesis. However, epinephrine also increases hepatic glucose output via glycogenolysis. In addition, they have opposite effects on insulin-mediated glucose uptake in peripheral tissue. Green + symbol indicates stimulation. Red X symbol indicates inhibition. such a relationship is seen in both the liver and extrahepatic tissues. In addition, norepinephrine increases the quantity of gluconeogenic precursors available in the liver. Infusion of norepinephrine causes the liver to shift from net output to net uptake of lactate (a gluconeogenic precursor), which increases gluconeogenesis [Cryer 1980]. Furthermore, norepinephrine stimulates lipolysis, which increases the substrate available for gluconeogenesis, resulting in increases in plasma glycerol levels while mediating the peripheral clearance of lactate. However, at lower plasma concentrations, norepinephrine's effect on glucose concentration is negligible [Connolly 1991].

It is important to note that norepinephrine's effect on gluconeogenic precursors and hepatic gluconeogenesis only attains kinetics sufficient for systemic alterations in glucose levels when high, neurotransmitterlike concentrations are achieved. These effects are independent of changes in the concentration of insulin and glucagon. However, despite the many mechanisms by which norepinephrine can affect glycemic control, the alterations in glycogenolysis caused by norepinephrine make an insignificant contribution to overall hepatic glucose production [Connolly 1991]. Although norepinephrine does have stimulatory effects on the availability of gluconeogenic precursors and can enhance the ability of the liver to convert these precursors to glucose, measurable downstream effects only occur at extremely high concentrations of this catecholamine.

\section{Norepinephrine's Interaction with Insulin and Peripheral Glucose Uptake}

In the absence of insulin, norepinephrine stimulates the uptake of glucose into peripheral tissues. In the presence of norepinephrine, glucose oxidation (the major mechanism of glucose metabolism) is increased $700 \%$, whereas lipogenesis is inhibited by $72 \%$ under these same conditions [Ebner 1987].

In the presence of insulin, norepinephrine's effects on glucose uptake and utilization are enhanced when compared to the isolated effects of norepinephrine alone. In an experiment using rat-derived brown adipocytes, there was a $200 \%$ increase in total glucose metabolism that occurred in the presence of norepinephrine alone. A combined infusion of norepinephrine and insulin yielded a 500\% increase in total glucose metabolism [Ebner 1987]. Marette et al additionally showed that norepinephrine acted to potentiate insulin's effect on the transport of glucose as evidenced by a leftward shift in the insulin dose-response curve while tracking the kinetics of transport in the presence of both insulin and norepinephrine compared to insulin alone [Marette 1989]. Thus, in stark contrast to the interplay between insulin and epinephrine, norepinephrine potentiates the effects of insulin and enhances the ability of peripheral tissue to take up and utilize glucose.

\section{COMPARISON OF EPINEPHRINE AND NOREPINEPHRINE IN HYPERGLYCEMIA}

Both epinephrine and norepinephrine are important in many phases of glucose homeostasis (see figure). Each hormone plays a role in hepatic glucose mobilization as well as gluconeogenesis. Epinephrine can significantly increase plasma glucose levels by promoting glycogenolysis in the 
liver. This effect is the main mechanism by which epinephrine increases plasma glucose. Epinephrine can also augment gluconeogenesis, and importantly, becomes the dominant mechanism of hyperglycemia after an hour of elevated epinephrine levels [Dufour 2009]. In contrast, norepinephrine primarily increases plasma glucose levels by mobilizing gluconeogenic precursors and enhancing the liver's ability to convert these substrates into glucose, although it does not seem to have a significant effect on promoting the breakdown of stored glycogen [Connolly 1991].

The effects of the interaction between insulin and epinephrine and between insulin and norepinephrine, at the level of the peripheral tissues, are also notably different. The 1950 study performed by Walaas et al and the 1984 studies by Sherwin et al demonstrated epinephrine's ability to attenuate the peripheral uptake of glucose mediated by insulin. These studies demonstrated that epinephrine significantly blunts the body's natural ability to decrease plasma glucose levels through its utilization at peripheral sites [Walaas 1950; Eigler 1979]. Norepinephrine however, has been observed to have a radically different interaction with insulin in the periphery. Experiments in rat adipocytes have demonstrated instead that norepinephrine potentiates insulin's ability to enhance glucose uptake by $250 \%$ [Ebner 1987].

In direct comparison, blood glucose changes with norepinephrine are less than that of epinephrine. In canine experiments, Connolly et al demonstrated that plasma glucose levels did not significantly change with low dose norepinephrine infusions. An infusion with higher norepinephrine levels caused the plasma glucose level to rise from $108 \mathrm{~g} / \mathrm{dL}$ to 159 $\mathrm{mg} / \mathrm{dL}$. Though this increase was significant, the authors note that the increase in glucose levels was not as great as that mediated by epinephrine [Connolly 1991; Stevenson 1991]. In models of chronic stress with prolonged hormone infusions, epinephrine plays an important role in sustained hyperglycemia, although circulating norepinephrine is less significant. In an epinephrine deficient infusion of stress hormones, there was a marked attenuation of both hepatic gluconeogenesis and glycogenolysis. In contrast, an epinephrine-deficient infusion resulted in no change on the stress hormone-induced alterations in glucose metabolism. Even more striking, glucose levels in the norepinephrine-deficient infusion were higher than in the control infusion, including all stress hormones. These findings suggest that, in a milieu of significant hyperglycemia, norepinephrine may in fact be protective, by limiting the hyperglycemia induced by other stress hormones [McGuinness 1997]. These findings provide strong evidence to suggest that norepinephrine plays a much smaller role in increasing plasma glucose levels than epinephrine.

\section{CONCLUSION}

Postoperative hyperglycemia is associated with a variety of adverse outcomes including increased mortality, length of stay, wound infections, and major cardiac events [Gerstein 2014; Ito 2014]. The catecholamines used to augment systemic blood pressure following cardiac surgery can have a significant influence on plasma glucose levels. There are significant differences in the effects of epinephrine and norepinephrine on hepatic glucose mobilization, peripheral utilization, and overall plasma glucose levels. Investigation into the mechanisms of action of each hormone and their systemic effects provide strong evidence that epinephrine is a much more potent mediator of hyperglycemia than is norepinephrine. This review suggests that in patients who require vasoactive agents following cardiac surgery, the use of norepinephrine results in better control of postoperative hyperglycemia as compared to epinephrine. We, therefore, recommend that norepinephrine be used in preference to epinephrine in patients with difficult-to-manage hyperglycemia who require vasoactive support following cardiac surgery.

\section{ACKNOWLEDGMENT}

Funding: This work was supported by the National Heart, Lung, and Blood Institute (grant T32 HL007849).

\section{REFERENCES}

Abel JJ, Crawford AC. 1897. On the blood-pressure-raising constituent of the suprarenal capsule. Bull Johns Hopkins Hosp 8:151-7.

Antonis A, Clark ML, Hodge RL, Molony M, Pilkington TR. 1967. Receptor mechanisms in the hyperglycaemic response to adrenaline in man. Lancet 1(7500):1135-7.

Bartnik M, Malmberg K, Norhammar A, Tenerz A, Ohrvik J, Rydén L. 2004. Newly detected abnormal glucose tolerance: an important predictor of long-term outcome after myocardial infarction. Eur Heart J 25(22):1990-7.

Bartnik M, Rydén L, Ferrari R, Malmberg K, Pyorala K, Simoons M, et al. 2004. The prevalence of abnormal glucose regulation in patients with coronary artery disease across Europe: the Euro Heart Survey on diabetes and the heart. Eur Heart J 25(21):1880-90.

Biggers DW, Myers SR, Neal D, et al. 1989. Role of brain in counterregulation of insulin-induced hypoglycemia in dogs. Diabetes 38(1):7-16.

Connolly CC, Steiner KE, Stevenson RW, et al. 1991. Regulation of glucose metabolism by norepinephrine in conscious dogs. Am J Physiol 261(6 Pt 1):E764-72.

Cryer PE. 1980. Physiology and pathophysiology of the human sympathoadrenal neuroendocrine system. N Engl J Med 303(8):436-44.

Deibert DC, DeFronzo RA. 1980. Epinephrine-induced insulin resistance in man. J Clin Invest 65(3):717-21.

Dufour S, Lebon V, Shulman GI, Petersen KF. 2009. Regulation of net hepatic glycogenolysis and gluconeogenesis by epinephrine in humans. Am J Physiol Endocrinol Metab 297(1):E231-5.

Ebner S, Burnol AF, Ferre P, de Saintaurin MA, Girard J. 1987. Effects of insulin and norepinephrine on glucose transport and metabolism in rat brown adipocytes: potentiation by insulin of norepinephrine-induced glucose oxidation. Eur J Biochem 170(1-2):469-74.

Edwards AV, Silver M. 1972. Comparison of the hyperglycaemic and glycogenolytic responses to catecholamines with those to stimulation of the hepatic sympathetic innervation in the dog. J Physiol 223(2):571-93.

Eigler N, Saccà L, Sherwin RS. 1979. Synergistic interactions of physiologic increments of glucagon, epinephrine, and cortisol in the dog: a model for stress-induced hyperglycemia. J Clin Invest 63(1):114-23. 
Furnary AP, Gao G, Grunkemeier GL, et al. 2003. Continuous insulin infusion reduces mortality in patients with diabetes undergoing coronary artery bypass grafting. J Thorac Cardiovasc Surg 125(5):1007-21.

Gerich JE. 1988. Glucose counterregulation and its impact on diabetes mellitus. Diabetes 37(12):1608-17. Lilly Lecture 1988.

Gerstein HC, Miller ME, Ismail-Beigi F, et al. 2014. Effects of intensive glycaemic control on ischaemic heart disease: analysis of data from the randomised, controlled ACCORD trial. Lancet 384(9958):1936-41.

Girish G, Agarwal S, Satsangi DK, Tempe D, Dutta N, Pratap H. 2014. Glycemic control in cardiac surgery: rationale and current evidence. Ann Card Anaesth 17(3):222-8.

Hatmann H, Beckh H, Jungermann K. 1982. Direct control of glycogen metabolism in the perfused rat liver by the sympathetic innervation. Eur J Biochem 123(3):521-6.

Hjemdahl P, Belfrage E, Daleskog M. 1979. Vascular and metabolic effects of circulating epinephrine and norepinephrine: concentrationeffect study in dogs. J Clin Invest 64(5):1221-8.

Ito N, Iwaya T, Ikeda K, et al. 2014. Hyperglycemia 3 days after esophageal cancer surgery is associated with an increased risk of postoperative infection. J Gastrointest Surg 18(9):1547-56.

Kvetnansky R, Sabban EL, Palkovits M. 2009. Catecholaminergic systems in stress: structural and molecular genetic approaches. Physiol Rev 89(2):535-606.

Lautt WW. 1983. Afferent and efferent neural roles in liver function. Prog Neurobiol 21(4):323-48.

Marette A, Bukowiecki LJ. 1989. Stimulation of glucose transport by insulin and norepinephrine in isolated rat brown adipocytes. Am J Physiol 257(4 Pt 1):C714-21.

McGuinness OP, Shau V, Benson EM, et al. 1997. Role of epinephrine and norepinephrine in the metabolic response to stress hormone infusion in the conscious dog. Am J Physiol 273(4 Pt 1) :E674-81.

Mulla I, Schmidt K, Cashy J, et al. 2014. Comparison of glycemic and surgical outcomes after change in glycemic targets in cardiac surgery patients. Diabetes Care 37(11): 2960-5.

Nobin A, Falck B, Ingemansson S, Järhult J, Rosengren E. 1977. Organization and function of the sympathetic innervation. Acta Physiol Scand Suppl 452:103-6.

Oliver G, Schäfer EA. 1895. The physiological effects of extracts of the suprarenal capsules. J Physiol 18(3):230-76

Reddy P, Duggar B, Butterworth J. 2014. Blood glucose management in the patient undergoing cardiac surgery: A review. World J Cardiol 6(11):1209-17.

Rui L. 2014. Energy metabolism in the liver. Compr Physiol 4(1):177-97.

Sacca L, Sherwin R, Felig P. 1978. Effect of sequential infusions of glucagon and epinephrine on glucose turnover in the dog. Am J Physiol 235(3):E287-90.

Sherwin RS, Sacca L. 1984. Effect of epinephrine on glucose metabolism in humans: contribution of the liver. Am J Physiol 247(2 Pt 1):E157-65.

Stevenson RW, Steiner KE, Connolly CC, et al. 1991. Dose-related effects of epinephrine on glucose production in conscious dogs. Am J Physiol 260(3 Pt 1):E363-70.

Takamine J. 1901. The isolation of the active principle of the suprarenal gland. J Physiol 27:29P-30P.

von Euler US. 1946. A specific sympathomimetic ergone in adrenergic nerve fibres (sympathin) and its relations to adrenaline and nor-adrenaline. Acta Physiol Scand 12(1):73-97.

Walaas O, Walaas E. 1950. Effect of epinephrine on rat diaphragm. J Biol Chem 187(2):769-76. 